\title{
Real and complex random neutrino mass matrices and $\theta_{13}$
}

\author{
Janusz Gluza ${ }^{1}$ and Robert Szafron ${ }^{1}$ \\ ${ }^{1}$ Institute of Physics, University of Silesia, Uniwersytecka 4, PL-40007 Katowice, Poland
}

\begin{abstract}
Recently it has been shown that one of the basic parameters of the neutrino sector, so called $\theta_{13}$ angle is very small, but quite probably non-zero. We argue that the small value of $\theta_{13}$ can still be reproduced easily by a wide spectrum of randomly generated models of neutrino masses. For that we consider real and complex neutrino mass matrices, also including sterile neutrinos. A qualitative difference between results for real and complex mass matrices in the region of small $\theta_{13}$ values is observed. We show that statistically the present experimental data prefers random models of neutrino masses with sterile neutrinos.
\end{abstract}

PACS numbers: 14.60.Pq; 02.10.Yn, 14.60.Lm

Since their first appearance in modern science neutrinos have played an important part in our understanding of the laws of particle physics. To see how important the weak interaction of neutrinos is in Nature lets just mention the mechanism in which the Sun is shining [1. It is also very well known that they influence evolution of the whole Universe as their tiny masses have an impact on the dynamics of the expansion of the Universe 2, 3. Without any doubt the investigation of the properties of these particles can reveal many interesting, hidden until now physical phenomena or explain many hypothetical ideas.

The theory of neutrino oscillations requires nonzero neutrino masses as well as nonzero neutrino mixing angles.

The T2K Collaboration recently announced that a new measurement 4 has yielded a nonzero $\theta_{13}$ for $\mathrm{CP}$ conserving case with $\delta_{C P}=0$ (results at $90 \%$ confidence level), i.e.

$$
\begin{aligned}
& 0.03 \leq \sin ^{2} 2 \theta_{13} \leq 0.28, \text { normal mass hierarchy, } \\
& 0.04 \leq \sin ^{2} 2 \theta_{13} \leq 0.34, \text { inverted mass hierarchy. }
\end{aligned}
$$

The Minos collaboration published similar results few days later [5. These results with nonzero $\theta_{13}$ are of primary importance for the future of particle physics [6, 7].

Present global fits of the data give [8] (at $1 \sigma$ C.L., 34])

$$
\begin{aligned}
7.05 \cdot 10^{-5} \mathrm{eV}^{2} & \leq \Delta m_{21}^{2} \leq 8.34 \cdot 10^{-5} \mathrm{eV}^{2} \\
2.07 \cdot 10^{-3} \mathrm{eV}^{2} & \leq \Delta m_{32}^{2} \leq 2.75 \cdot 10^{-3} \mathrm{eV}^{2} \\
0.39 & \leq \sin ^{2} \theta_{23} \leq 0.5 \\
0.291 & \leq \sin ^{2} \theta_{12} \leq 0.324 \\
0.008 & \leq \sin ^{2} \theta_{13} \leq 0.036 .
\end{aligned}
$$

There are many theoretical models which tune the space of possible neutrino parameters to get agreement with these experimental data. The new data from the T2K collaboration generated a new challenge for that. Frequently the pattern of a flavour symmetry is invoked, for recent theoretical adjustments of the nonzero $\theta_{13}$ mixing angle, see e.g. [10. Here we face the problem differently asking how natural are small, nonzero values of $\theta_{13}$.

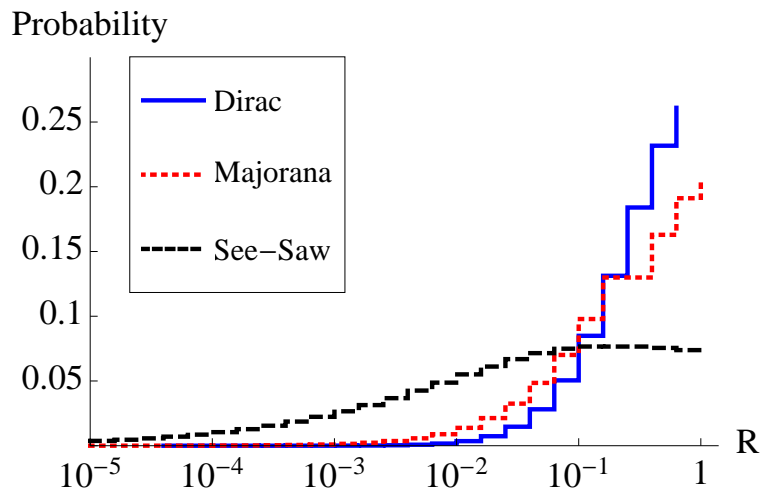

FIG. 1: Frequency probability for $R$ parameter obtained from $10^{7}$ randomly generated three dimensional different types of matrices. No constraints given by Eqs $3-7$ are applied.

Naturalness means that the neutrino mass matrices are generated randomly. Apart from neutrino physics, see e.g. [11, 17, random matrices came to be an eminent tool used in many different fields of science, including day-to-day life problems 18 .

Let us first consider a symmetric, three dimensional matrix with random elements in the range $[-1,1]$. Physically this case realizes Majorana type of neutrinos. Similarly, we consider also Dirac neutrinos (generated by general real random matrices) and so-called see-saw neutrinos with masses $m_{\nu}$ defined through relation $m_{D}^{T} M_{R}^{-1} m_{D}$ (here again matrices $m_{D}$ and $m_{R}$ are generated randomly in the range $[-1,1])$. Next, we apply the singular value decomposition theorem [19] to calculate the mixing matrix $U$ which diagonalizes the random matrices we started from. Using the standard parametrisation, we are able to link experimental mixing angles to the elements of matrix $U$, e.g. $\sin ^{2} \theta_{13}=\left|U_{13}\right|^{2}$.

In Fig, 1 we recover one of the results discussed already in [11, see Fig.1. Here $R=\Delta m_{21}^{2} / \Delta m_{32}^{2}$ is the ratio of the smallest to the next smallest values of differences of squared neutrino masses, $m_{1}<m_{2}<m_{3}$. Both in Fig 1 and next plots results are generated based on $10^{7}$ random mass matrices. Hall et al. approach has an advantage of 


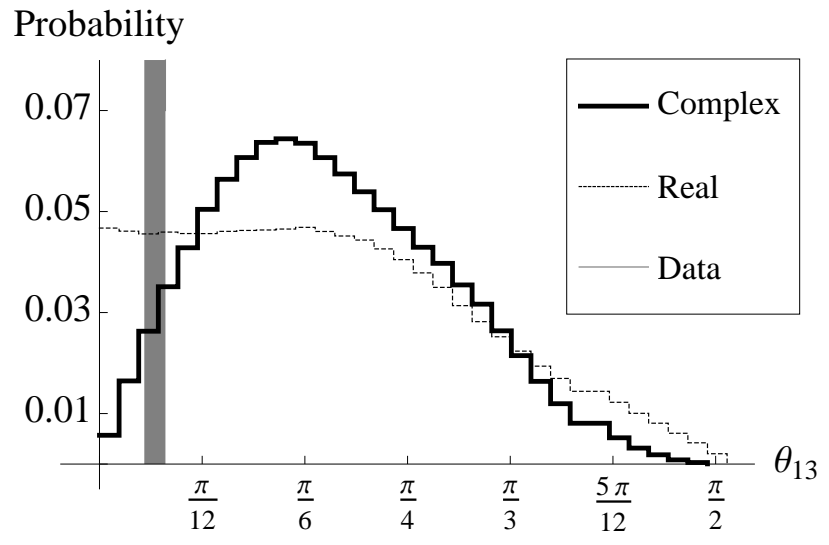

FIG. 2: Frequency probability for $\theta_{13}$ obtained from $10^{7}$ randomly generated real and imaginary three dimensional symmetric matrices (Majorana neutrinos). No constraints given by Eqs 3.6 are applied. Gray vertical band represents experimental values of $\theta_{13}, \mathrm{Eq} 7$ at the $1 \sigma$ level.

pure simplicity. A modified versions have been considered in many papers, see e.g. [12 16].

Let us note that the see-saw neutrino masses are trivially not well defined if elements of $M_{R}$ approach zero in relation $m_{D}^{T} M_{R}^{-1} m_{D}$, which can happen as elements of $M_{D}$ and $M_{R}$ are generated in the $[-1,1]$ range. Thus we will consider only Dirac and Majorana type of neutrinos. To disentangle these two types of neutrinos is of great importance for neutrino physics [20. If we connect the mechanism of generation of their tiny masses with some heavy states, this issue starts to be important for high energy colliders like $e^{+} e^{-}$[21, 22], $e^{-} e^{-}$[23] and LHC 24.

We can extend this discussion to the case where elements of the neutrino mass matrix involve complex phases (random numbers with modulus in the interval $[0,1]$ and a phase in a range $[0 ; 2 \pi])$. It might seem that real and imaginary matrices should produce qualitatively the same results: situation differs only as an additional degree of freedom (phase) enters in each entry of the mass matrix. However, this is not true, as in fact they differ. This change does not affect the shapes of the eigenvalues distributions in a significant way but other observables distributions like elements of the mixing matrix may change. This is exactly the case for $\theta_{13}$.

Fig 2 shows the results of numerical predictions for $\theta_{13}$, additional constraints given by Eqs 36 are not applied. We can see that the real and imaginary random matrices behave qualitatively differently in vicinity of small $\theta_{13}$ and real matrices fit better. That frequency probability of random complex matrices tends to zero with $\theta_{13} \rightarrow 0$ can be understood in the following way. For real matrices CP complex phase is zero or $\pi$ and CP is not violated when $\theta_{13}=0$ and therefore there is no direct restriction on values of $\theta_{13}$. Reversing the argument, if CP symme-

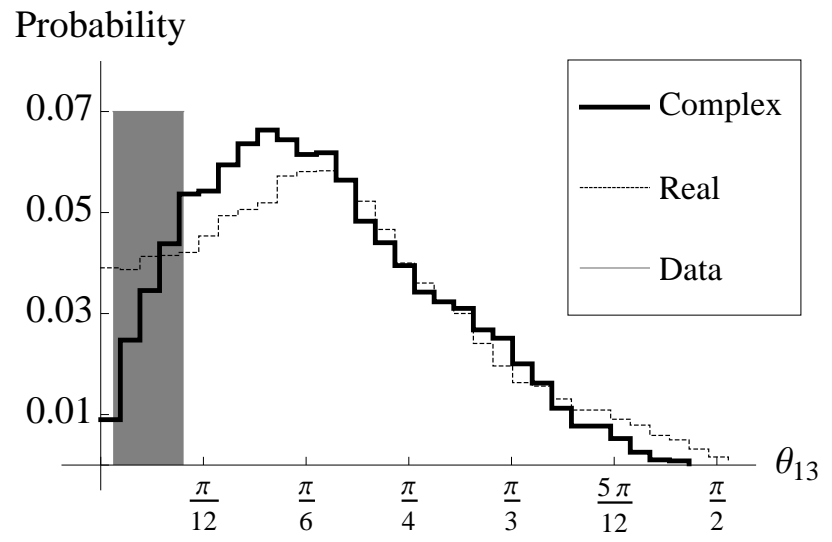

FIG. 3: Frequency probability for $\theta_{13}$ obtained from $10^{7}$ randomly generated real and imaginary three dimensional symmetric matrices (Majorana neutrinos). Constraints by Eqs 3 . 6 are applied at the $3 \sigma$ level. Gray vertical band represents experimental values of $\theta_{13}, \mathrm{Eq} 7$ at the $3 \sigma$ level.

try is violated (which is the case for randomly generated complex mass matrices) then $\theta_{13}$ must be different from zero. We found that frequency probability for the physical CP violating phase $\delta$ which emerges from randomly generated mass matrices is constant (so this is a trivial plot and we do not show it here. From pure mathematics (a probability measure) follows that in such case a probability to get $\delta$ equal to zero or $\pi$ is zero, so probability distributions should tend to zero for $\theta_{13} \rightarrow 0$, what can be seen on histograms, see solid line in Fig. 2, If Eqs 3 36 are applied, a number of mass matrices which cover the experimentally interesting values of $\theta_{13}$ decreases. For instance, using $1 \sigma$ cuts in Eqs 36 , only 4886 real and 4686 complex matrices remain out of initial $10^{7}$ random matrices. Using $3 \sigma$ cuts we are left with 31853 real and 29141 complex matrices, see Fig.3. Comparing between Dirac and Majorana cases more mass matrices in Majorana case survive the cuts. The main conclusion remains however the same as in the case where no cuts Eqs 36 are applied: there are more matrices which reproduce experimental data for real matrices and complex matrices do not condense in the experimentally interesting region.

It is interesting that the last experimental data implies the presence of a fourth sterile neutrino [25 28]. Usually the so called $3+1$ and $3+2$ models of sterile neutrinos are considered [35]. We then inspect the mass matrices of dimensions four and five. Here we focus on an element $\left|U_{13}\right|^{2}$ and define an effective angle such that $\sin ^{2} \theta_{13}=\left|U_{13}\right|^{2}$, so we can compare the results obtained from models with different number of neutrinos.

In a case of more than three dimensional mass matrices relations among the elements of the mixing matrix and experimentally defined mixing angles are more involved, therefore we will consider this case without cuts driven by Eqs 37 and without a discussion of the im- 


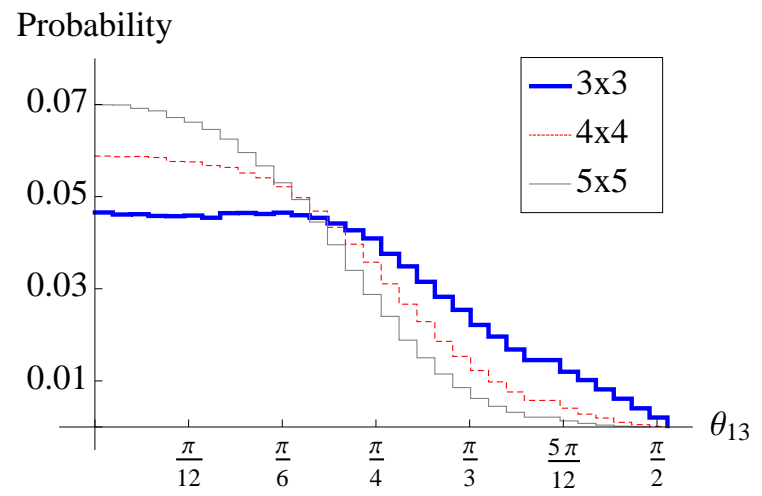

FIG. 4: Shifts in the direction of small values of $\theta_{13}$ with increasing number of sterile neutrinos. No constraints given by Eqs 3 are applied.

pact of additional mixing matrix parameters of four and five dimensional mass matrices. We proceed as in the previous case and generate random matrices and then calculate the mixing matrix. In Fig 4 we show results for Majorana (symmetric) real matrices of different dimensions. We observe that the more neutrinos we have the bigger number of random matrices reproduce the experimental value of the $\theta_{13}$ parameter. Similar results give Dirac neutrinos.

We have no clear explanation why the probability of getting small values for $\theta_{13}$ grows with dimensionality of matrices. There is a mathematical law of Wigner 29. connected with real symmetric random matrices which states that their eigenvalues accumulate around zero (Wigner's semicircle law [30] is exact in the limit of infinite dimensions of matrices). To our knowledge, there is no relation connecting the distributions of the values of elements of eigenvectors with the dimensionality of real symmetric matrices.

Nonetheless, let us try to understand the result we obtained. In the anarchical model of neutrino masses, when no cuts on parameters are applied, mixing angles have very similar probability distributions. This is because every element of the mass matrix has exactly the same probability distribution. Therefore, if the dimension of the mass matrix grows, the unitarity of the mixing matrix constrains sums of squared modulus of mixing matrix elements, and an average value of each of elements of the mixing matrix will decrease with increasing dimensionality. This forces the probability distribution to take higher values for small angles as the dimension grows, a kind of pattern obtained in Fig 4

We can make a step further and consider for the first time implications of nonzero $\theta_{13}$ on values of elements $\left|U_{14}\right|$ and $\left|U_{24}\right|$ in the $3+1$ sterile model when the constraint Eq 7 for $\theta_{13}$ is applied. (Without cuts, probability distributions for $\left|U_{14}\right|$ and $\left|U_{24}\right|$ are practically the same as for $\theta_{13}$ in Fig, 2, as the just discussed equal-

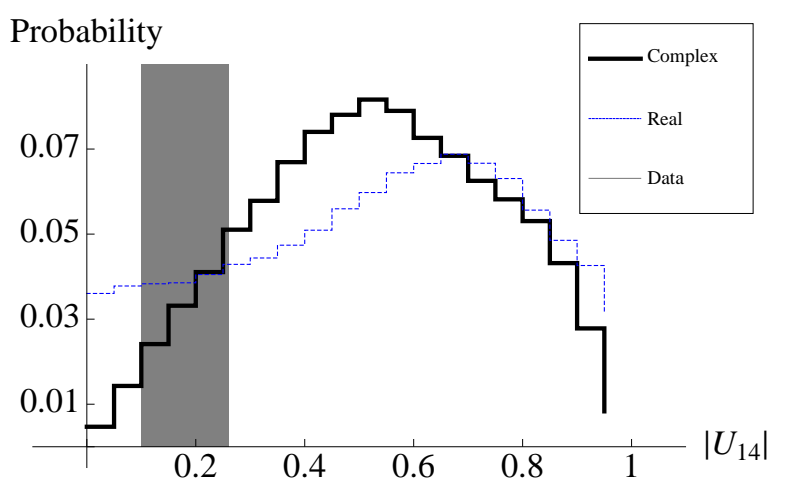

FIG. 5: Probability distributions for $\left|U_{14}\right|$ elements of the neutrino mixing matrix when the constraint Eq7 for $\theta_{13}$ is applied. The gray vertical band stands for $3 \sigma$ values which follow from global fits 32 .

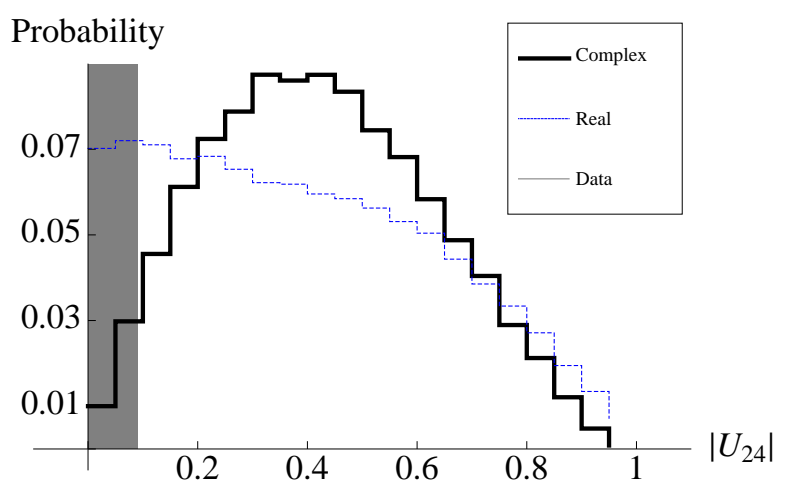

FIG. 6: Probability distributions for $\left|U_{24}\right|$ elements of the neutrino mixing matrix when the constraint Eq.7 for $\theta_{13}$ is applied. The gray vertical band stands for upper bounds derived by MINOS [33].

distribution argument suggests.) It is interesting that analyses of recent experiments predict non-zero values for them [31, 32]. In Fig. 5 and Fig. 6 the plots are given for real and complex neutrino Majorana mass matrices (Dirac patterns are very similar). We can see that probability distributions for both real and complex cases reach a maximum at larger values of $\left|U_{14}\right|\left(\left|U_{14}\right|_{\max } \simeq 0.7\right.$ for a real case, $\left|U_{14}\right|_{\max } \simeq 0.58$ for a complex case) when compared with $\left|U_{24}\right|\left(\left|U_{24}\right|_{\max } \simeq 0.1\right.$, real case; $\left|U_{14}\right|_{\max } \simeq 0.4$, complex case). This is in agreement with fits discussed in 32$]\left(\left|U_{\mu 4}\right|^{2}<\left|U_{e 4}\right|^{2}\right.$ for both low and high energy neutrino experimental fits). In Fig 5 we can see that the most frequent values of $\left|U_{14}\right|$ do not coincide with its experimentally preferable region, though in this experimental region they can also be substantial.

More involved analyses including other experimental constraints and various mass hierarchies in $3+1$ and $3+2$ models go beyond this Brief Report and are left for a future work.

In conclusion, though random matrices can not solve 
fundamental problems in neutrino physics, they generate intriguing hints on the nature of neutrino mass matrices.

We would like to thank Marek Gluza and Radomir Sevillano for useful discussions and careful reading of the manuscript. Work supported in part by the Research Executive Agency (REA) of the European Union under the Grant Agreement number PITN-GA-2010-264564 (LHCPhenoNet), by the Polish Ministry of Science under grant No. N N202 064936 and National Science Centre.

[1] John N. Bahcall, How the Sun Shines, http://nobelprize.org/nobel_prizes/physics/articles/fusion/

[2] D. W. Sciama, Nature 348 (2003) 617.

[3] G. Dvali, Nature 432 (2004) 567.

[4] T2K Collaboration, K. Abe et al., Phys.Rev.Lett. 107 (2011) 041801.

[5] MINOS Collaboration, P. Adamson et al., Phys.Rev.Lett. (2011).

[6] Mohapatra, R. N., Pal, P. B., Massive Neutrinos in Physics and Astrophysics, World Scientific, 2004. Third Edition, Lecture Notes in Physics, Vol. 72.

[7] C. Giunti, C. W. Kim, Fundamentals of Neutrino Physics and Astrophysics, Oxford University Press, Oxford, UK, 2007.

[8] G. L. Fogli, E. Lisi, A. Marrone, A. Palazzo and A. M. Rotunno, Phys. Rev. D 84 (2011) 053007.

[9] T. Schwetz, M. Tortola and J. W. F. Valle, New J. Phys. 13 (2011) 109401

[10] E. Ma and D. Wegman, Phys.Rev.Lett. 107 (2011) 061803.

[11] L. J. Hall, H. Murayama, and N. Weiner, Phys.Rev.Lett. 84 (2000) 2572-2575.

[12] F. Vissani, Phys. Lett. B 508 (2001) 79 arXiv:hep$\mathrm{ph} / 0102236$.

[13] G. Altarelli, F. Feruglio and I. Masina, JHEP 0301 (2003) 035 hep-ph/0210342.

[14] A. de Gouvea and H. Murayama, Phys. Lett. B 573 (2003) 94

[15] S. -F. Ge, D. A. Dicus and W. W. Repko, Phys. Lett. B
702 (2011) 220 arXiv:1104.0602 [hep-ph]].

[16] S. -F. Ge, D. A. Dicus and W. W. Repko, arXiv:1108.0964 [hep-ph].

[17] B. Dziewit, K. Kajda, J. Gluza, and M. Zralek, Phys.Rev. D74 (2006) 033003.

[18] Deift, P.,Universality for mathematical and physical systems, arXiv:0603038 [math-ph].

[19] SVD Nash, J. C. The Singular-Value Decomposition and Its Use to Solve Least-Squares Problems

Ch. 3 in Compact Numerical Methods for Computers: Linear Algebra and Function Minimisation, 2nd ed. Bristol, England: Adam Hilger, pp. 30-48, 1990.

[20] B. Kayser, F. Gibrat-Debu, and F. Perrier, World Sci.Lect.Notes Phys. 25 (1989) 1-117.

[21] J. Gluza and M. Zralek, Phys. Rev. D 48 (1993) 5093; ibid. 55 (1997) 7030; J. Gluza, J. Maalampi, M. Raidal and M. Zralek, Phys. Lett. B 407 (1997) 45.

[22] F. del Aguila and J. Aguilar-Saavedra, JHEP 0505 (2005) 026.

[23] J. Gluza and M. Zralek, Phys.Lett. B362 (1995) 148-154; J. Gluza and M. Zralek, Phys. Rev. D 52 (1995) 6238.

[24] F. del Aguila, J. Aguilar-Saavedra, and R. Pittau, JHEP 0710 (2007) 047.

[25] C. Giunti, arXiv:1106.4479 [hep-ph].

[26] C. Giunti and M. Laveder, Phys. Rev. D 84 (2011) 073008

[27] O. Yasuda, JHEP 1109 (2011) 036.

[28] C. Giunti and M. Laveder, arXiv:1109.4033 [hep-ph].

[29] E. Wigner, Ann. of Math. 62 (1955) 548-564.

[30] WolframMathWorld, http://mathworld.wolfram.com/WignersSemicircleLaw.html

[31] C. Giunti and M. Laveder, Phys. Rev. D 84 (2011) 073008; ibid, D 84 (2011) 093006.

[32] C. Giunti and M. Laveder, Phys. Lett. B 706 (2011) 200.

[33] P. Adamson et al. [The MINOS Collaboration], Phys. Rev. D 81 (2010) 052004.

[34] Fits in 9] differs slightly, but the exact values of parameters are not the main issue here.

[35] For the see-saw mechanism, there are additional righthanded singlets with a much higher mass scale. But it is not the only possible scenario. Still, light sterile neutrinos are possible. That is why we extend the anarchy neutrino mass model of Haba et al. directly to higher dimensions. Moreover, this can be a kind of an effective mass matrix which originates from more complicated mechanisms. 